Supporting Information

\title{
Proteolytic activation of Bacillus thuringiensis Cry2Ab through a belt-and-braces approach
}

\author{
Lian $\mathrm{Xu}{ }^{\star}, \#$, Zhi-Zhen Pan ${ }^{\star}, \#$, Jing Zhang ${ }^{\dagger}$, Bo Liu $*$, Yu-Jing Zhu *,*, \\ Qing-Xi Chen ${ }^{\dagger}, *$
}

$\dagger$ State Key Laboratory of Cellular Stress Biology, Key Laboratory of the Ministry of Education for Coastal and Wetland Ecosystems, School of Life Sciences, Xiamen University, Xiamen 361005, China.

* Agricultural Bio-Resources Institute, Fujian Academy of Agricultural Sciences, Fuzhou 350003, China.

${ }^{\#}$ The authors contribute equally in this work.

*Corresponding author.

(Qing-Xi Chen) Tel: 0592-2185487/13459256685, Fax: 0592-2185487, E-mail: chenqx@xmu.edu.cn.

(Yu-Jing Zhu) E-mail: zyjingfz@ 163.com. 

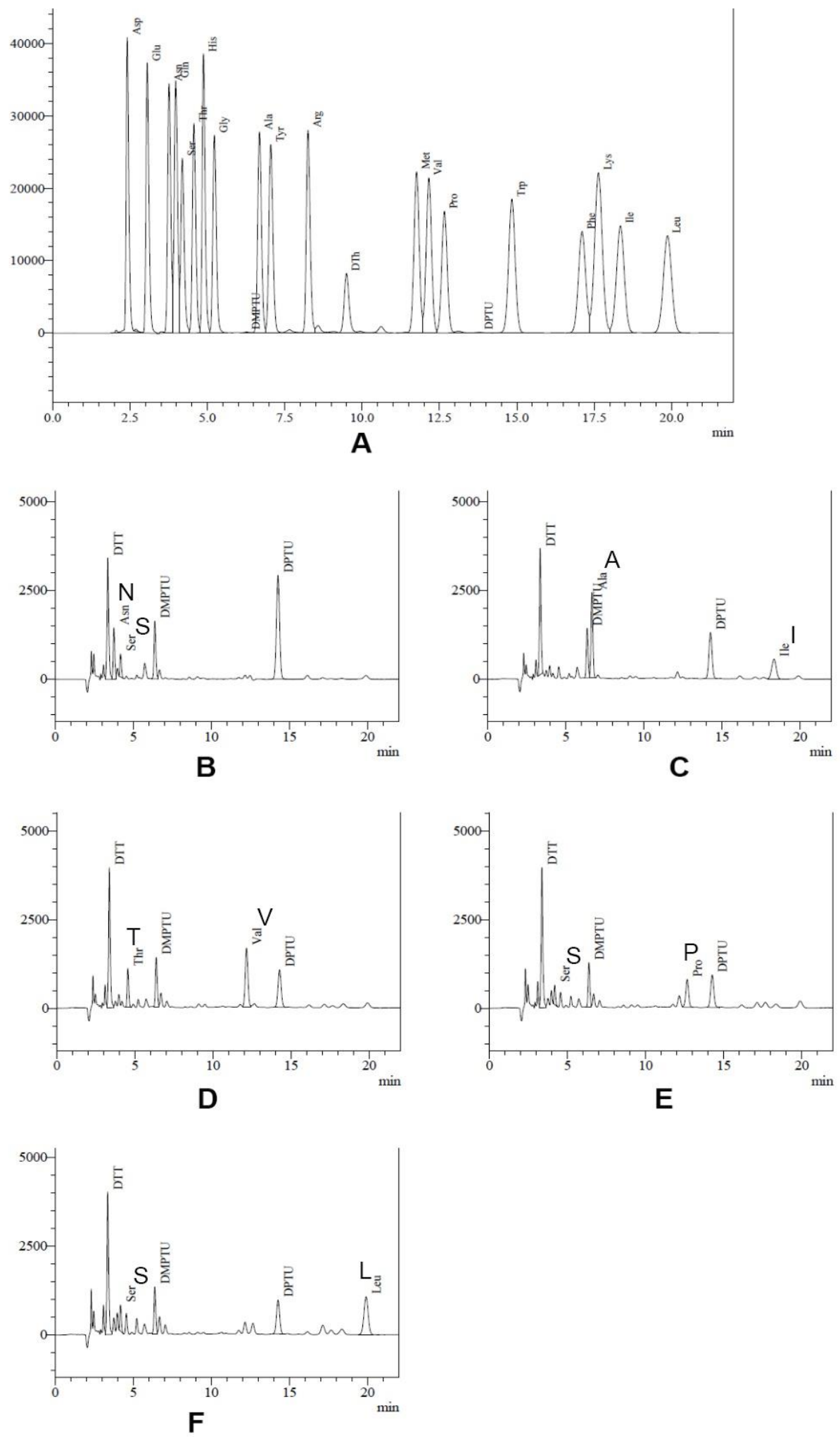
Fig S1. N-terminal sequencing identified cleavage-sites of Cry2Ab processed by PxMJ.

(A) Spectrum of 19 PTH-amino acids standards; (B)-(F) N-terminal amino acid identification of Cry2Ab activated-toxin. 